\title{
Beitrag zur Frage Oser und Kames
}

\section{Von Gerhard Kelle r, Ibbenbüren-Hannover. Mit 4 Textabbildungen}

Utber die Frage der Entstehung der Oser und Kames besteht heute insofern Übereinstimmung, als beide Gruppen als fluvioglaziale Bildungen aus dem letzten Abschnitt einer Eiszeit aufgefaßt werden, in dem das aktiv bewegte Inlandeis in Toteisareale zerfiel. Da in Nordwestdeutschland die Ablagerungen der Saaleund der Weichseleiszeit oberflächenformend auftreten, sind aus beiden Zeiten derartige fluvioglaziale Absätze vorhanden. Die Entscheidung, welche Form von ihnen vorliegt, muß sowohl von dem äußeren morphologischen Bild als auch von der inneren Textur ausgehen, bedarf im einzelnen aber oft eingehender Feststellungen an guten Aufschlüssen. Zur Verfeinerung der Methodik soll im folgenden unter Verwendung von Beobachtungen aus dem nördlichen Westfalen und dem anschließenden Niedersachsen versucht werden, auf systematische Fragen bei den Osern und Kames einzugehen.

\section{Morphologische Gesichtspunkte}

Im Landschaftsbild erscheinen die Oser als sich oft auf $10-20 \mathrm{~km}$ erstreckende, sehr schmale, z. T. gratartige Rücken, die einem mehr als $60 \mathrm{Jahre}$ alten Vergleich WAHNSCHAFFE's (1890) entsprechend künstlich aufgeschütteten Eisenbahndämmen sehr ähnlich sind. WoLDSTEDT (1929) berichtete von solchen Osern in Jungmoränenlandschaften und wies auf das modellartige Beispiel des von Bärtling (1905) beschriebenen Neuenkirchener Oses an der mecklenburgischlauenburgischen Grenze hin. Ein fast gradliniges Teilstück von $650 \mathrm{~m}$ Länge hat bei einer Sohlenbreite von $50 \mathrm{~m}$ eine Höhe von $16 \mathrm{~m}$. Der Kamm hat nur eine Breite von etwa $1 \mathrm{~m}$. Im Längsprofil steigen die Rücken der Oser mäßig auf und ab.

Neben geraden Strecken finden sich Windungen und Bögen, die an Flußläufe erinnern. Nebenoser münden zuweilen in den Hauptos ein. Im Altmoränengebiet der Saaleeiszeit können die mehr oder weniger geschlossenen Kieswälle in Einzelhügel aufgelöst sein, besonders wenn eine abdeckende Grobkiesschicht lückenhaft ist und die unterlagernden Feinsandschichten erodiert werden konnten. Die Kames sind ursprünglich schon breiter und flacher als die Oser. Einzelhügel, die $3-6 \mathrm{~km}$ lang und $200-500 \mathrm{~m}$ breit werden können, finden sich neben Kleinformen von noch nicht $100 \mathrm{~m}$ Länge und $30 \mathrm{~m}$ Breite. Diese Kleinformen pflegen schwarmartig aufzutreten, sind dann mehr oder weniger parallel angeordnet und hintereinander gestaffelt.

Wegen der breiten Rückenform ist daran gedacht worden, diese auf die Alterung vor allem der saaleeiszeitlichen Bildungen zurückzuführen. Es zeigt sich aber an westfälischen Beispielen, daß unter der oberen $1-1,5 \mathrm{~m}$ mächtigen entschichteten Zone in den tiefer folgenden Schichten die breite Rückenform primär angelegt ist. Weiterhin erscheint es fraglich, ob bei der schnellen Aufnahme- und Versickerungsfähigkeit der meist groben Sedimente eine seitliche Verlagerung bzw. ein seitliches Fließen in größerem Umfang statthaben konnte. Auch sind senkrecht stehende, in Richtung des Gefälles nicht verzerrte Brodeltöpfe auf saaleeiszeitlichen Kames in Westfalen ein Hinweis darauf, daß keine Erniedrigung der Kuppen, ja noch nicht einmal eine seitliche Bewegung in der Hangrichtung, seit mindestens dem Periglazial der Weichseleiszeit erfolgte (KELLER 1951).

Ähnliche Längen wie die Oser können auch die Kames erreichen. Dort handelt es sich dann um eine Kette aneinandergereihter primär entstandener Einzelhügel. Diese sind gegenseitig versetzt und bilden insgesamt einen Kameszug, 
dessen einzelne Glieder mehrere Kilometer lang werden können. Nach morphologischen Gesichtspunkten besteht somit offensichtlich die Möglichkeit einer Trennung. Konvergenzen im morphologischen Bild zwischen den Osern und Kames treten bei älteren Bildungen aus der Saaleeiszeit auf, wenn z. B. ein von der Abtragung angegriffener Os in eine Kette von kürzeren Einzelhügeln aufgelöst ist, die an sich bei Kames als typisch gelten können.

\section{Texturelle Gesichtspunkte}

Für die innere Textur hat Woldstedt (1929) angegeben, daß die Oser aus horizontal geschichteten Sanden und Kiesen mit Schräg- und Kreuzschichtung bestehen. WAHNSCHAFFE (1890) hat derartige Lagerungsverhältnisse beschrieben und abgebildet. In neuerer Zeit kennzeichnet Beringer (1951) die Textur der Oser als aus waagerechten Schichten bestehend. Auch im folgenden soll diese Eigenart als typisch für den inneren Bau der Oser angesehen werden. Die Schichtung streicht aus dem Hang heraus in das Freie. Nach WaHnschaffe tritt ein ganz schwaches Einfallen in den äußersten Teilen der Querprofile auf, das aber auf nachträgliches Rutschen am Gehänge zurückzuführen ist.

Nach Angaben im älteren Schrifttum soll die Textur der Kames ebenfalls in horizontaler Schichtung bestehen. Diese Feststellung ist nach Beobachtungen an westfälischen Kames bedingt richtig. Es trifft wohl zu, daß die Kames in ihrem zentralen Teil längs der Rückenlinie horizontal geschichtet sind, wobei Schrägund Kreuzschichtung sehr oft vorkommt. Nach den Seiten zu biegt die Schichtung aber ab. Die wirkliche Textur der Kames tritt erst bei großen Querschnitten in Erscheinung, indem sich die einzelnen Schichten in Schalen mantelförmig übereinander legen. Auf diese Erscheinung hat Woldstedt (1929) hingewiesen und sie mit einer Beeinflussung der Schichtlagerung durch das Forttauen des seitlich begrenzenden Eises in Verbindung gebracht.

Als Kames sind daher gewölbeartig hochgestellte und durch eine konzentrisch-schalig angeordnete Textur ausgezeichnete Sand- und Kieshügel anzusehen, bei denen außerdem die Schichtung gleichsinnig mit der äußeren Hangneigung einfällt. Im älteren Schrifttum der achtziger und neunziger Jahre wird öfters von diesem gewölbeartigen Aufbau berichtet, dabei aber kein Unterschied zwischen Osern und Kames im heutigen, stärker differenzierenden Sinne gemacht. Bis in die zwanziger Jahre wurden die Kames teilweise auch noch als Endmoränen aufgefaßt (WaHnschaffe \& Schucht 1921). Auch im neueren geomorphologischen Schrifttum (MACHATSCHEK 1949), das sich hauptsächlich auf die Schilderung der Oser beschränkt, wird die im Querprofil parallel zur Hügeloberfläche verlaufende Schichtung für die Oser in Anspruch genommen. Jedoch weiß Wahnschaffe (1890) bereits zu berichten, daß die Schichten der Kames oft stark gewölbt sind und eine Art „Übergußstruktur“ zeigen.

Sowohl bei Osern als auch bei Kames kann die Textur durch die Einlagerung eines Kernes aus älteren Diluvialschichten (sehr oft Geschiebelehm, auch Staubeckensedimente) verwickelter werden. Der kernhaltige Typ wird bei den Osern nach KorN (1910) als Aufpressungsos bezeichnet. Der von unten eingedrungene Kern hat eine dach-oder gewölbeartige Aufrichtung der primär waagerecht liegenden Sand- und Kiesbänke des Os bewirkt. Die mit einem Kern ausgestatteten Kames, die im weiteren zweckmäßig als „Kernkames“ bezeichnet werden sollen, haben mit den Aufpressungsosern lediglich die Kernfüllung gemeinsam. Die Schrägstellung und gewölbeartige Aufrichtung der Sand- und Kiesschichten 
der Kames beruht aber im Gegensatz zu den Aufpressungsosern auf einer anderen Voraussetzung, da die Aufrichtung auch dort vorhanden ist, wo ein älterer Sedimentkern fehlt.

\section{Genetische Gesichtspunkte}

Ausgangspunkt für die genetische Deutung der Oser und Kames ist der heutige morphologische und texturelle Befund. Abgesehen von der Theorie der inglaziären Entstehung kann die Theorie der supraglaziären neben der subglaziären die größte Wahrscheinlichkeit für das Zutreffen ihrer Deutung in Anspruch nehmen. Die Anschauung der supraglaziären Entstehung geht auf HoLsT (1876) zurück. Auf der Oberfläche des Toteises schnitten sich die Schmelzwässer in Flußbetten ein. Die mitgeführten Sedimente stammen aus dem Eis selbst, das bereits stark zurückgetaut war und den mehr liegenden Teil freigab, der sich

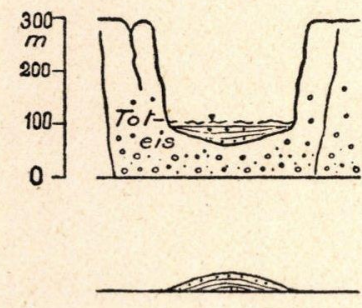

a
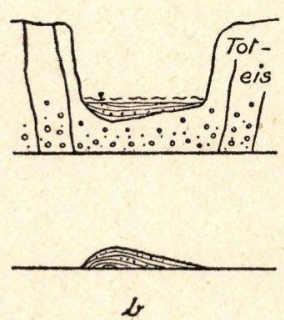
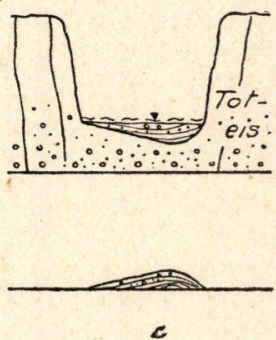

Abb. 1. Bildung der Kames in supraglaziären Flußbetten. Obere Reihe bei der Entstehung, untere Reihe die heutigen Hügelformen. Querschnitte: a) symmetrische Form, b) und c) unsymmetrische Formen in Abhängigkeit von der Lage des ehemaligen Stromtsriches. Nicht überhöht.

durch die Führung größerer Schuttmassen auszeichnete. Nach dem Fortschmelzen der mehr oder weniger schüsselförmigen Eisbetten (Abb. 1a-1c) legte sich zuerst der mächtigere Mittelteil bzw. die Füllung des Stromstriches auf den Untergrund. Die seitlich auskeilenden Sedimentzungen folgten und senkten sich herab.

Die ursprünglich horizontale Schichtung geht dadurch in gewölbeartige Texturen über. In kleinerem Bereich treten Abbrüche an den Seiten auf, die schließlich infolge weiteren Absinkens noch antithetisch überkippt sein können (s. Abb. 3d). Je nach der Lage des ehemaligen Stromstriches als der Zone größter Mächtigkeit entstehen symmetrische oder unsymmetrische Hügelformen mit einseitig steilerer Böschung (Abb. 1b und 1c). Diesen Vorgängen verdanken die breiteren und im Einzelteil kurzen Kames als Ausfüllungen breiterer Lücken im Toteis ihre Entstehung. Die Hintereinanderreihung der Einzelkames zu Kameszügen spiegelt den Verlauf der einzelnen Flußsysteme wieder. Die Flußsysteme folgen ihrer ersten Anlage entsprechend dem Gefälle der Toteisoberfläche und können in ihrer Richtung von der ehemaligen Bewegungsrichtung des Inlandeises verschieden sein. Zwischen den einzelnen Strömungswannen liegen primär sedimentfreie Untiefen, die heute als Geländedepressionen erkennbar sind.

Bei der subglaziären Entstehung der Oser sitzen die Ossedimente der Sohle des Gletschers unmittelbar auf (Abb. 2a). Der schmale Tunnelquerschnitt läßt nach dem Forttauen einen ebengeschichteten Hügel zurück (Abb. 2c), an dessen unterem Teil sich die Hänge anböschen können. Wenn auch die subglaziäre Entstehung der Oser, wie z. B. in Eistunneln oft als die wesentlichere erkannt wird 
(WoLDSTEDT 1950), so kann auf die supraglaziäre Bildungsweise nicht verzichtet werden, soweit die Oser Aufpressungsoser darstellen. Die Aufpressungsoser setzen den frei zur Wirkung kommenden Druck von aufsteigenden Eiswänden (Abb. 2b) voraus, die nur in Verbindung mit steilen Schluchten oder klammartigen Ausräumungen vorgelegen haben können, wie auch voN BüLow (1940)

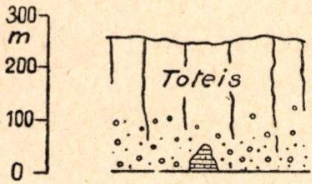

a

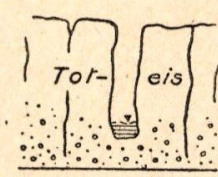

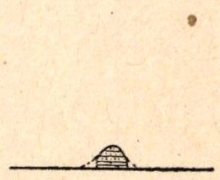

Abb. 2. Bildung von Osern. a) subglaziär (in Eistunneln); b) supraglaziär in klammartigen Schluchten; c) der heutige Os. Nicht überhöht.

darstellte. Über der Tunnelform subglaziärer Entstehung ist der Druck wegen der Überlagerung durch die massive tragende Eismasse verteilter und damit wesentlich schwächer, so daß er nicht ausreicht, die meist 10 und $20 \mathrm{~m}$ hohen Aufpressungen des Untergrundes zu erzeugen. Außerdem stehen die schlauchartigen Gebilde unter hydrostatischem Überdruck von etwa 10 bis 20 atü. Auf die hierbei wichtige Eismächtigkeit wird anschließend zurückzukommen sein.

Entsprechend den Darstellungen der Abb. 3 u. 4 wird die Bildung der Kernkames und der Aufpressungsoser mit der supraglaziären Entstehungsweise in Verbindung gebracht. Mit genügender Zuverlässigkeit kann auf Grund von Belastungszahlen bei Tonen, die durch das Inlandeis der Saaleeiszeit vorbelastet waren, die maximale Toteismächtigkeit für das Gebiet am Nordwestende des Teutoburger Waldes mit $250-300 \mathrm{~m}$ angegeben werden. Bei streifenförmiger Belastung längs der Eiswände der Schmelzwasserläufe treten an der Basis des Toteises Drucke von $22-27 \mathrm{~kg} / \mathrm{cm}^{2}$ auf. Solange die supraglaziären Flüsse noch wenig in das Toteis eingeschnitten sind, erfolgt keine Aufpressung des Liegen-
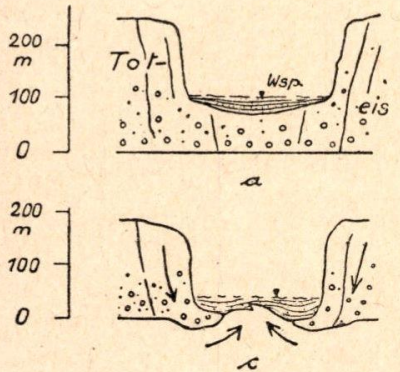
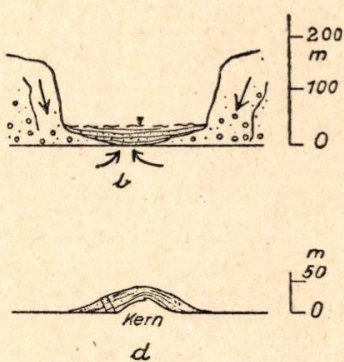

Abb. 3. Bildung eines Kernkames. a) normale Entstehung eines Kames; b) das Flußbett schneidet das Liegende des Toteises an; c) der Eisdruck wird aktiv; d) der heutige Kernkame (links antithetische Verwerfungen). Nicht überhöht.

den. Erst bei dem Vordringen der Erosion in die unterste Zone des Toteises fehlt der Widerstand und der Eisdruck wirkt sich in dem Empordrücken von Sedimentkeilen aus dem Liegenden aus.

Der in einer breiten Rinne fließende Schmelzwasserstrom der Abb. 3a hat noch unter seinem Bett etwa $70 \mathrm{~m}$ mächtiges Toteis. Die fortschreitende Erosion schafft das folgende Bild, in dem festgehalten ist, wie die Unterikante des Bettes gerade das Liegende des Toteises anschneidet (Abb. 3b). Der spätestens zu die- 
sem Zeitpunkt aktiv werdende Eisdruck preßt das Liegende als Kern in die Sedimentfüllung des Kamesbettes hinein (Abb. 3c), und es entsteht das Texturbild eines Kernkames (Abb. 3d). Wenn auch eine teilweise Schrägstellung der Kamessedimente durch diesen Vorgang mit verursacht sein kann, so ist diese ganz wesentlich durch das Forttauen der gewölbten Eisunterlage bedingt, wie die kernfreien Kames sehr deutlich vor Augen führen (Abb. 1).

Die Entstehung von Aufpressungsosern im Sinne von KonN knüpft an schmale Lücken im Eis an. Oft ist bei ihnen auch die bestätigte Ansicht vertreten, daß diese Hohlräume in der Bewegungsrichtung des Inlandeises verlaufende Radialspalten im Toteis sind. Die Spalten begünstigen die supraglaziäre Entstehung der Oser. Die Schmelzwässer flossen mit freiem Wasserspiegel durch die Kanäle ab. Während bei nicht bis in die Sohle des Eises eindringenden Spalten oder bei

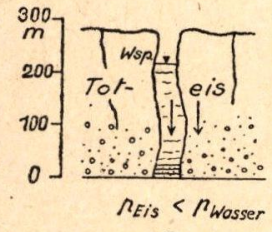

a

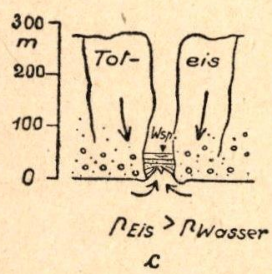

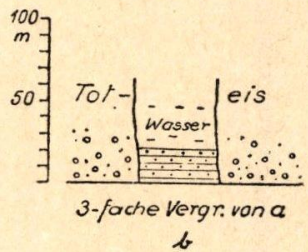

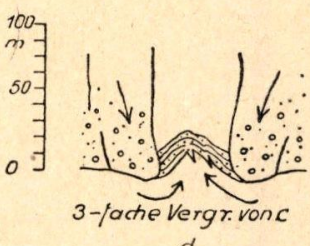

d

Abb. 4. Bildung von Aufpressungsosern. a) von Wasser erfüllte Eisspalte mit hohem Wasserdruck einschl. Sedimenten ohne Aufpressung; b) Vergrößerung von a) zeigt horizontalgeschichtete Ossedimente; c) der Wasser- und Sedimentdruck in der Eisspalte ist dem Eisdruck gegenüber gering, so daß Aufpressung eintritt, wie unter d) nochmals vergrößert gezeigt wird. Alles nicht überhöht.

mit Wasser mehr oder weniger ausgefüllten Spalten (Abb. 4a) eine Aufpressung des Liegenden nicht erfolgt, kann diese bei dem Schwinden der Eissohle oder dem Ablaufen des Wasserinhaltes auftreten. Wie die Wasserfüllung einer Spalte einerseits die Entstehung von Aufpressungsosern verhindert und wie andererseits der durch Anschneiden der Sohle freiwerdende Belastungsdruck sie bewirkt, zeigen die Abbildungen $4 \mathrm{a}-4 \mathrm{~d}$, wobei hinzuzufügen ist, daß die Abbildungen $4 \mathrm{~b}$ und $4 \mathrm{~d}$ jeweils die gut dreifachen Vergrößerungen der nebenstehenden Abbildungen $4 \mathrm{a}$ und $4 \mathrm{c}$ darstellen.

Ergebnis e

Zur Frage der Unterscheidung von Osern und Kames als fluvioglaziale Bildungen aus dem Toteisabschnitt einer weichenden Eiszeit hat WoldsTEDT (1929) die Frage aufgeworfen, ob es berechtigt ist, die beiden Formen als abgrenzbare Typen aufzufassen, oder ob sie das gleiche sind und damit eine Trennung überflüssig ist. Unter Betonung des sehr unterschiedlichen Bildes der schmalen, langgestreckten eisenbahndammartigen Oser und der im Einzelglied kurzen und breiten Kames, die oft auch gesellig auftreten, hat WoLDSTEDT vom morphologischen Standpunkt ausgehend die Trennung bejaht. 
Bei der Erörterung, wie weit auch die texturellen Eigenheiten für die Unterscheidung der Oser und Kames herangezogen werden können, ist darauf hinzuweisen, daß für die Oser die horizontale Schichtung als charakteristisch bezeichnet wurde. Dem ist aber hinzuzufügen, daß sie bei diesen sicher nicht als der Regelfall zu gelten hat. Doch dürfte bei ihrem Auftreten auf eine Osbildung geschlossen werden können. Bei den morphologisch typischen Kames findet sich dagegen stets die gewölberatige konzentrisch-schalige Textur.

Nach dem älteren Schrifttum, in dem allerdings die Unterscheidung von Osern und den so gut wie nicht beachteten und bekannten Kames vielfach nicht berücksichtigt wurde, wird die gewölbeartige Textur z. T. auch bei Osern angeführt. In der Tat ist heute, auch unter Berücksichtigung einer schärferen begrifflichen Formulierung, eine große Anzahl morphologisch typischer Oser mit derartiger Textur bekannt.

Zweifellos liegen in den Aufpressungsosern und den Kernkames texturelle Konvergenzerscheinungen verschiedener genetischer Entstehung vor, die das exakte Bild verwischen. Auch werden, wie im nördlichen Westfalen, Einzelhügel angetroffen, die zunächst den Eindruck einer Kette von Kameshügeln erwecken, sich dann aber nach ihrer horizontal geschichteten Textur als Teile eines in Einzelstücke aufgelösten saaleeiszeitlichen Oses zu erkennen geben.

In der Trennung der morphologischen Typen Oser und Kames spiegelt sich teilweise ihre genetische Verschiedenheit wieder, die in dem Gegensatz subglaziärer und supraglaziärer Bildungsweise zum Ausdruck kommt. Auch die texturelle Verschiedenheit horizontal geschichteter Osersedimente aus Eistunneln und gewölbeartig aufgerichteter Kamessedimente aus fortgeschmolzenen Flußbetten der Eisoberfläche erscheint hierdurch erklärt. Doch dürfte der eindeutige morphologische Osertyp in genetischer Hinsicht neben den subglaziären auch supraglaziäre Entstehungsmerkmale vereinigen, wie sich am Beispiel der Aufpressungsoser nachweisen läßt.

\section{Angeführte Literatur}

BärtLING, R.: Der Os am Neuenkirchener See an der mecklenburgisch-lauenburgischen Landesgrenze. - Jb. preuß.-geol. LA. 26, S. 15-25. 1905.

Beringer, C. Chr.: Geologisches Wörterbuch. - Stuttgart (F. Enke) 1951.

voN BüLow, K.: Erdgeschichte und Landschaftsgestaltung im Kreise Stolp in Pommern. - Stolp (Pom.), O. Eulitz, 1930.

Holst, N. D.: Om de glaziala rullstens-aosarne. - Geol. Fören. Förh. Stockholm 3, S. 97-112. 1876 (zitiert nach WAHNSChaFfe 1892).

KELLER, G.: Die Deutung des Kiessandrückens in Laer-Heide und Laer-Höhe (Bez. Osnabrück) als Kame. - Neues Jb. Geol. u. Paläont., Mh., S. 353-362, Stuttgart 1951.

Konn, J.: Erl. Bl. Marienfließ der geol. Spezialk. von Preußen 1:25000 - Berlin 1910.

Machatschek, Fr.: Geomorphologie. - Leipzig (Teubner) 1949.

WahnschafFe, F.: Über einen Grandrücken bei Lubasz. - Jb. preuß. geol. L.A. f. 1890, S. 277-288. Berlin 1892.

Wahnschaffe, F. \& Schucht, Fr.: Geologie und Oberflächengestaltung des Norddeutschen Flachlandes. - Stuttgart (F. Engelhorn Nachf.). 1921.

Woldstedt, P.: Das Eiszeitalter. - Stuttgart (F. Enke). 1929. - Norddeutschland und angrenzende Gebiete im Eiszeitalter. - Stuttgart (K. F. Koehler). 1950. 